H. Yoshida

Nagoya Math. J.

Vol. 87 (1982), 41-57

\title{
JULIA DIRECTIONS OF ENTIRE FUNCTIONS OF SMOOTH GROWTH
}

\author{
H. YOSHIDA
}

\section{§1. Introduction}

Let $f(z)$ be entire i.e. analytic in the finite whole plane $Z$. The order of $f(z)$ is defined as

$$
\rho=\varlimsup_{r \rightarrow \infty} \frac{\log ^{+}\left(\log ^{+} M(r, f)\right)}{\log r}
$$

where $M(r, f)=\max _{|z|=r}|f(z)|$. A ray $\chi(\theta)=\left\{z=r \cdot e^{i \theta}: 0<r<+\infty\right\}$ is called a Julia direction of $f(z)$ if, in any open sector containing the ray, $f(z)$ takes all values of $Z$, with at most one finite exceptional value, infinitely often.

We can guess that the smoothness of growth of $M(r, f)$ causes simple boundary behaviors of $f(z)$. In this paper, we exemplify this fact, by picking up two kinds of smoothness conditions.

The following problem comes into question: Let $f(z)$ be an entire function of order less than $\frac{1}{2}$ and let $\chi(\theta)$ be any ray. Either is $\chi(\theta)$ a Julia direction of $f(z)$ or is $f(z)$ convergent to $\infty$ as $|z| \rightarrow+\infty$ on some sector containing $\chi(\theta)$ ? So, we shall prove in Theorem 2 that if we assume the smoothness of growth of $M(r, f)$ : if there is a constant $\mu, \mu<\frac{1}{2}$, such that

$$
\frac{\log M\left(x_{0} \cdot r, f\right)}{\log M(r, f)} \leqq x_{0}^{\mu} \quad\left(r \geqq r_{0}\right)
$$

for some $x_{0}, x_{0}>1$, and $r_{0}$, this fact is true. Theorem 1 is the preliminary result for this theorem.

Further, we shall show in Theorem 3 that, under the assumption of the stronger smoothness condition:

Received November 2, 1979.

Revised May 2, 1981. 


$$
\log M(2 \cdot r, f) \sim \log M(r, f), \quad(r \rightarrow \infty)
$$

a Julia direction $\chi(\theta)$ of $f(z)$ is characterized as the ray $\chi(\theta)$ for which $\theta$ is a limit point of the set

$$
Z(f)=\left\{\arg z_{n}: f\left(z_{n}\right)=0\right\} .
$$

Hence, according to Hayman [9, p. 143], it follows that all Julia directions of entire functions $f(z)$ satisfying the condition

$$
\log M(r, f)=O\left(\log ^{2} r\right) \quad(r \rightarrow \infty)
$$

are the directions corresponding to the limit points of the set $Z(f)$. Hayman [9, p. 130] remarked that any entire function satisfying (B) has order 0 . An example will be given to show that any entire function of order 0 has not always this property.

By using this Theorem 3, we shall give an example of an entire function $f(z)$ for which any non-empty closed set is precisely the set of Julia directions of $f(z)$. This generalizes an example of Anderson and Clunie [2].

The author wishes to acknowledge with grateful thanks the help of Prof. W. K. Hayman.

\section{$\S 2$. The boundary behaviour of entire functions}

In the following, the spherical derivative of a meromorphic function $f(z)$ is defined by

$$
\rho(f(z))=\frac{\left|f^{\prime}(z)\right|}{1+|f(z)|^{2}} .
$$

We denote the set $\left\{z:\left|z-z_{0}\right|<\varepsilon\left|z_{0}\right|\right\}$ by $D\left(z_{0}, \varepsilon\right)$ and the sector $\{z:|\arg z-\theta|$ $<\varepsilon\}$ by $V(\theta, \varepsilon)$.

Lemma 1 (Clunie and Hayman [4, p. 125]). Let $f(z)$ be regular in $\left|z-z_{0}\right| \leqq \delta$ and satisfy $|f(z)| \geqq 1$ there. If $\left|f\left(z_{1}\right)\right|=1$ for some $z_{1}$ with $\left|z_{1}-z_{0}\right|=\delta$, then for some $z$ on the segment joining $z_{0}$ to $z_{1}$ we have

$$
\rho(f(z)) \geqq \frac{\log \left|f\left(z_{0}\right)\right|}{10 \cdot \delta \cdot \log 2} .
$$

LEMMA 2. Let $f(z)$ be an entire function and let $\delta$ be a constant, $0<\delta<1$. If $\left\{z_{n}\right\},\left|z_{n}\right| \rightarrow \infty$, is a sequence such that 


$$
\left|f\left(z_{n}\right)\right| \rightarrow \infty
$$

and $f(z)$ does not converge to $\infty$ as $|z| \rightarrow+\infty$ on the set $\bigcup_{n} D\left(z_{n}, \delta\right)$, then there is a sequence $\left\{\xi_{k}\right\},\left|\xi_{k}\right| \rightarrow \infty, \xi_{k} \in \bigcup_{n} D\left(z_{n}, \delta\right)$, satisfying

$$
\lim _{k \rightarrow \infty}\left|\xi_{k}\right| \cdot \rho\left(f\left(\xi_{k}\right)\right)=+\infty .
$$

Proof. By the assumption, we can find a subsequence $\left\{\boldsymbol{z}_{n_{k}}\right\}$ of $\left\{\boldsymbol{z}_{n}\right\}$ and a sequence $\left\{\zeta_{k}\right\},\left|\zeta_{k}\right| \rightarrow \infty, \zeta_{k} \in D\left(z_{n_{k}}, \delta\right)$, for which

$$
\left|f\left(\zeta_{k}\right)\right| \leqq K
$$

where $K$ is a constant, $K \geqq 1$. Put $\delta_{k}=\operatorname{dis}\left(S, z_{n_{h}}\right)$, where $S=\{z:|f(z)|$ $\leqq K\}$ and $\operatorname{dis}(A, B)$ denotes the distance between $A$ and $B$. Then, we have

$$
\delta_{k} \leqq\left|\zeta_{k}-z_{n_{k}}\right| \leqq \delta\left|z_{n_{k}}\right| \quad(k=1,2,3, \cdots) .
$$

Now, consider the function

$$
g(z)=\frac{f(z)}{K} .
$$

From Lemma 1 applied to $g(z)$, we see that there is a sequence $\left\{\xi_{k}\right\},\left|\xi_{k}-z_{n_{k}}\right|$ $\leqq \delta_{k}$, such that

$$
\rho\left(g\left(\xi_{k}\right)\right) \geqq \frac{\log \left|g\left(z_{n_{k}}\right)\right|}{10 \cdot \delta_{k} \cdot \log 2} \quad(k=1,2,3, \cdots) .
$$

Since

$$
\rho(f(z)) \geqq-\frac{1}{K} \cdot \rho(g(z))
$$

and

$$
\left|\xi_{k}\right| \geqq(1-\delta) \cdot\left|z_{n_{k}}\right| \quad(k=1,2,3, \cdots),
$$

from (1), we finally get from (1) and (2) that

$$
\left|\xi_{k}\right| \cdot \rho\left(f\left(\xi_{k}\right)\right) \geqq \frac{(1-\delta) \cdot\left\{\log \left|f\left(z_{n_{k}}\right)\right|-\log K\right\}}{10 \cdot \delta \cdot K \cdot \log 2} \quad(k=1,2,3, \cdots)
$$

which gives us the conclusion.

LEMMA 3. Let $\theta, \rho_{1}$ and $\rho_{2}$ be constants satisfying $0 \leqq \theta<2 \pi, 0<\rho_{1}<1$, $0<\rho_{2}<1$ and let $z_{1}, z_{2}$ be any numbers on $\chi(\theta)$. If the circles $D\left(z_{1}, \rho_{1}\right)$ 
and $D\left(z_{2}, \rho_{2}\right)$ intersect, then the angle which is subtended at the origin by the chord connecting the points of intersection is dependent only on $t=z_{2} / z_{1}$, $\rho_{1}$ and $\rho_{2}$.

Proof. We can see from easy calculation that $(Y / X)^{2}$ is the function dependent on $t, \rho_{1}$ and $\rho_{2}$, where $(X, Y)$ denotes the coordinate of the points of intersection of both circles.

Lemma 4 (Lehto [11, Theorem 3]). Let $f(z)$ be meromorphic in $R<|z|<\infty$. If, for some sequence $\left\{\xi_{k}\right\},\left|\xi_{k}\right| \rightarrow \infty$

$$
\lim _{k \rightarrow \infty}\left|\xi_{k}\right| \cdot \rho\left(f\left(\xi_{k}\right)\right)=+\infty,
$$

then $f(z)$ assumes every value infinitely often with at most two exceptions of values in the extended plane on the set $\cup_{k} D\left(\xi_{k}, \varepsilon\right)$ for each fixed $\varepsilon>0$.

We now state and prove

THEOREM 1. Let $f(z)$ be an entire function and $\chi(\theta)(0 \leqq \theta<2 \pi)$ be a ray on which there exist a sequence $\left\{\boldsymbol{z}_{n}\right\},\left|\boldsymbol{z}_{n}\right|<\left|\boldsymbol{z}_{n+1}\right|,\left|\boldsymbol{z}_{n}\right| \rightarrow \infty$, and a constant $M$, satisfying

$$
\left|\frac{z_{n+1}-z_{n}}{z_{n}}\right|<M
$$

and

$$
\lim _{n \rightarrow \infty}\left|f\left(z_{n}\right)\right|=+\infty
$$

Then, $\chi(\theta)$ is a Julia direction of $f(z)$ or $f(z)$ is convergent to $\infty$ as $|z| \rightarrow$ $+\infty$ on some sector containing $\chi(\theta)$.

Proof. First of all, suppose that $f(z)$ does not converge to $\infty$ as $|z|$ $\rightarrow+\infty$ in the set $\bigcup_{n} D\left(z_{n}, \varepsilon\right)$ for any $\varepsilon>0$. Then, by Lemma 2 , for any $\varepsilon, 0<\varepsilon<1$, we can find a sequence $\left\{\zeta_{k}\right\}, \zeta_{k} \in D\left(z_{n_{k}}, \varepsilon\right)$, such that

$$
\lim _{k \rightarrow \infty}\left|\zeta_{k}\right| \cdot \rho\left(f\left(\zeta_{k}\right)\right)=+\infty
$$

Lemma 4 shows that $f(z)$ assumes every value of $Z$ infinitely often with at most one exception in the set $V(\theta, \pi \varepsilon)$ and hence $\chi(\theta)$ is a Julia direction of $f(z)$.

So, suppose that $f(z)$ converges to $\infty$ as $|z| \rightarrow+\infty$ in the set $\bigcup_{n} D\left(z_{n}, \varepsilon\right)$ for some $\varepsilon>0$, and denote by $E_{1}$, the set of these $\varepsilon$ 's. We put 


$$
\rho_{1}=\sup _{\varepsilon \in E_{1}} \varepsilon
$$

If $\rho_{1}>1$, we have $\bigcup_{n} D\left(z_{n}, \varepsilon\right)=Z$ for some $\varepsilon \in E_{1}, \varepsilon>1$, and hence we get evidently the conclusion. So, we suppose that $0<\rho_{1} \leqq 1$. Take the sequence $\left\{\boldsymbol{z}_{n}^{(2)}\right\}, \boldsymbol{z}_{n}^{(2)} \in \chi(\theta)$, satisfying

$$
\left|\boldsymbol{z}_{n}^{(2)}\right|=\left|\boldsymbol{z}_{n}\right| \cdot\left(1+\frac{1}{2} \cdot \rho_{1}\right) \quad(n=1,2,3, \cdots) .
$$

By using the fact that

$$
\left|f\left(z_{n}^{(2)}\right)\right| \rightarrow \infty \quad(n \rightarrow \infty),
$$

we repeat the same argument. If $f(z)$ does not converge to $\infty$ as $|z| \rightarrow$ $+\infty$ in the set $\bigcup_{n} D\left(z_{n}^{(2)}, \varepsilon\right)$ for any $\varepsilon>0$, we can also conclude that $\chi(\theta)$ is a Julia direction of $f(z)$. In the case that $f(z)$ converges to $\infty$ as $|z| \rightarrow$ $+\infty$ in the set $\bigcup_{n} D\left(z_{n}^{(2)}, \varepsilon\right)$ for some $\varepsilon>0$, denote by $E_{2}$ the set of these $\varepsilon$ 's and put

$$
\rho_{2}=\sup _{\varepsilon \in E_{2}} \varepsilon .
$$

Then we can suppose that $0<\rho_{2} \leqq 1$. Again, take the sequence $\left\{\boldsymbol{z}_{n}^{(3)}\right\}, \boldsymbol{z}_{n}^{(3)}$ $\epsilon \chi(\theta)$, satisfying

$$
\left|z_{n}^{(3)}\right|=\left|z_{n}^{(2)}\right| \cdot\left(1+\frac{1}{2} \cdot \rho_{2}\right)=\left|z_{n}\right| \cdot\left(1+\frac{1}{2} \cdot \rho_{1}\right) \cdot\left(1+\frac{1}{2} \cdot \rho_{2}\right) \quad(n=1,2,3, \cdots) .
$$

Repeat this process over and over until we get either the conclusion that $\chi(\theta)$ is a Julia direction of $f(z)$ or the conclusion

$$
\prod_{i=1}^{N}\left(1+\frac{1}{2} \cdot \rho_{i}\right)>M+1
$$

at some step $N$. In the case that (3) happens, we can easily show from Lemma 3 that $f(z)$ converges to $\infty$ as $|z| \rightarrow+\infty$ on the set $V(\theta, \alpha)$ for some $\alpha>0$.

Now, suppose that these processes are continued infinitely. Then, we have

$$
\prod_{i=1}^{\infty}\left(1+\frac{1}{2} \cdot \rho_{i}\right) \leqq M+1
$$

Since $f(z)$ does not converge to $\infty$ as $|z| \rightarrow+\infty$ on the set $\bigcup_{n} D\left(z_{n}^{(i)}, 2 \cdot \rho_{i}\right)$ for each $i$ satisfying $\rho_{i}<\frac{1}{2}$, Lemma 2 gives a sequence $\left\{\xi_{k}^{(i)}\right\},\left|\xi_{k}^{(i)}\right| \rightarrow \infty$ $(k \rightarrow \infty), \xi_{k}^{(i)} \in \bigcup_{n} D\left(z_{n}^{(i)}, 2 \cdot \rho_{i}\right)$, such that

$$
\lim _{k \rightarrow \infty}\left|\xi_{k}^{(i)}\right| \cdot \rho\left(f\left(\xi_{k}^{(i)}\right)\right)=+\infty
$$


From the fact $\rho_{i} \rightarrow 0$ and Lemma 4 , we can conclude that $\chi(\theta)$ is a Julia direction of $f(z)$. Thus, we complete the proof.

To prove Theorem 2, we need the following property (Lemma 7) of entire functions $f(z)$ for which $\log M(r, f)$ satisfies the smoothness condition (A).

LEMma 5. Let $x_{0}, x_{0}>1, \mu, \mu \geqq 0, r_{0}$ and $R, r_{0}>R$, be constants. If $h(r)$ is a positive, non-decreasing function defined on the interval $R<r<$ $+\infty$ and satisfies the condition:

$$
\frac{h\left(x_{0} \cdot r\right)}{h(r)} \leqq x_{0}^{\mu \prime} \quad\left(r \geqq r_{0}\right),
$$

then

$$
\frac{h(x \cdot r)}{h(r)} \leqq x_{0}^{\mu} \cdot x^{\mu} \quad\left(r \geqq r_{0}\right)
$$

for any $x, x \geqq x_{0}$, and

(ii) for any $\alpha, \alpha>\mu$,

$$
\int_{r}^{\infty} \frac{h(t)}{t^{1+\alpha}} d t \leqq S\left(x_{0}: \alpha, \mu\right) \cdot \frac{h(r)}{r^{\alpha}} \quad\left(r \geqq r_{0}\right),
$$

where

$$
S\left(x_{0}: \alpha, \mu\right)=\frac{x_{0}^{\alpha}-1}{\alpha\left(x_{0}^{\alpha}-x_{0}^{\mu}\right)} \cdot x_{0}^{\mu} .
$$

Proof. Take any $x \geqq x_{0}$ and choose an integer $p$ such that $x_{0}^{p} \leqq x<$ $x_{0}^{p+1}$. Then,

$$
h(x \cdot r) \leqq h\left(x_{0}^{p+1} \cdot r\right) \leqq\left(x_{0}^{p+1}\right)^{\mu} \cdot h(r) \leqq x_{0}^{\mu} \cdot x^{\mu} \cdot h(r) \quad\left(r \geqq r_{0}\right) .
$$

This gives (i).

Since

$$
h\left(x_{0}^{i+1} \cdot r\right) \leqq\left(x_{0}^{\mu}\right)^{i+1} \cdot h(r), \quad\left(r \geqq r_{0}\right) \quad(i=0,1,2, \cdots)
$$

we have

$$
\begin{aligned}
\int_{r}^{\infty} \frac{h(t)}{t^{1+\alpha}} d t & \leqq \sum_{i=0}^{\infty} h\left(x_{0}^{i+1} \cdot r\right) \cdot \int_{x_{0}^{i} \cdot r}^{x_{0}^{i+1} \cdot r} \frac{1}{t^{1+\alpha}} d t \\
& \leqq \frac{x_{0}^{\mu}}{\alpha} \cdot\left[1-\frac{1}{x_{0}^{\alpha}}\right] \cdot \frac{h(r)}{r^{\alpha}} \cdot \sum_{i=0}^{\infty}\left(x_{0}^{\mu-\alpha}\right)^{i}=S\left(x_{0}: \alpha, \mu\right) \cdot \frac{h(r)}{r^{\alpha}} \quad\left(r \geqq r_{0}\right)
\end{aligned}
$$


Thus, (ii) is obtained.

Lemma 6. (Denjoy [5] and Kjellberg [10, p. 17-18].) Let $f(z)$ be an entire function of order $\mu, 0 \leqq \mu<\frac{1}{2}$, and $f(0)=1$. Then, for any $\alpha, \mu<$ $\alpha<\frac{1}{2}$,

$$
\begin{array}{r}
r^{\alpha} \cdot \int_{r}^{\infty}[\log m(t, f)-(\cos \pi \alpha) \cdot \log M(t, f)] \cdot \frac{d t}{t^{1+\alpha}}>\frac{1-\cos \pi \alpha}{\alpha} \cdot \log M(r, f) \\
(0<r<+\infty),
\end{array}
$$

where $m(t, f)=\min _{|z|=t}|f(z)|$.

LEMMA 7. Let $f(z)$ be an entire function for which $f(0)=1$ and $\log M(r, f)$ satisfies the condition (A): there is constant $\mu, \mu<\frac{1}{2}$, such that

$$
\frac{\log M\left(x_{0} \cdot r, f\right)}{\log M(r, f)} \leqq x_{0}^{\mu} \quad\left(r \geqq r_{0}\right)
$$

for some $x_{0}, x_{0}>1$, and $r_{0}$. Then, for any $\alpha, \mu<\alpha<\frac{1}{2}$, there exists a constant $k$ such that for some $t$ in any interval $(r, k \cdot r)\left(r \geqq r_{0}\right)$

$$
\log m(t, f)>\cos \pi \alpha \cdot \log M(t, f) .
$$

Proof. First of all, we have

$$
\begin{aligned}
& r^{\alpha} \cdot \int_{x \cdot r}^{\infty}[\log m(t, f)-(\cos \pi \alpha) \cdot \log M(t, f)] \cdot \frac{d t}{t^{1+\alpha}} \\
& \quad \leqq r^{\alpha}(1-\cos \pi \alpha) \cdot \int_{x \cdot r}^{\infty} \frac{\log M(t, f)}{t^{1+\alpha}} d t \\
& \quad \leqq r^{\alpha} \cdot(1-\cos \pi \alpha) \cdot S\left(x_{0}: \alpha, \mu\right) \cdot x_{0}^{\mu} \cdot x^{\mu-\alpha} \cdot \log M(r, f) \\
& \quad\left(x \geqq x_{0}, r \geqq r_{0}\right)
\end{aligned}
$$

from Lemma 5 in which $h(r)=\log M(r, f)$. Thus, since we see from (i) of Lemma 5 that $f(z)$ has at most order $\mu$, we get

$$
\begin{array}{r}
r_{\alpha} \cdot \int_{r}^{x \cdot r}[\log m(t, f)-(\cos \pi \alpha) \cdot \log M(t, f)] \cdot \frac{d t}{t^{1+\alpha}} \\
>(1-\cos \pi \alpha) \cdot\left[\frac{1}{\alpha}-S\left(x_{0}: \alpha, \mu\right) \cdot x_{0}^{\mu} x^{\mu-\alpha}\right] \cdot \log M(r, f) \\
\left(x \geqq x_{0}, r \geqq r_{0}\right)
\end{array}
$$

from Lemma 6 . Here, if we take a $k, k \geqq x_{0}$, such that

$$
\frac{1}{\alpha}-S\left(x_{0}: \alpha, \mu\right) \cdot x_{0}^{\mu} \cdot k^{\mu-\alpha}>0,
$$


the right-hand side of the inequality in which $x$ is replaced with $k$ is always positive for all $r \geqq r_{0}$ and hence the left-hand side is positive. Thus, we obtain the conclusion.

Now, we have

TheOREM 2. Let $f(z)$ be an entire function for which $\log M(r, f)$ satisfies the smoothness condition (A) for some $\mu, x_{0}$ and $r_{0}$, where $\mu<\frac{1}{2}$ and $x_{0}>1$. Then, for any ray $\chi(\theta)(0 \leqq \theta<2 \pi), \chi(\theta)$ is a Julia direction of $f(z)$ or $f(z)$ is convergent to $\infty$ as $|z| \rightarrow+\infty$ on some open sector containing $\chi(\theta)$.

Proof. It is evident that we can confine ourselves to the case $f(0)=1$. If we denote by $t_{n}$ such a $t$ of the interval $\left(k^{n} \cdot r_{0}, k^{n+1} \cdot r_{0}\right)(n=0,1,2, \cdots)$ in Lemma 7, we have

$$
\left|\frac{t_{n+1}-t_{n}}{t_{n}}\right| \leqq \frac{k^{n+2} \cdot r_{0}-k^{n} \cdot r_{0}}{k^{n} \cdot r_{0}}=k^{2}-1
$$

Thus, we see that the sequence $\left\{t_{n} \cdot e^{i \theta}\right\}$ for any fixed $\theta(0 \leqq \theta<2 \pi)$ is a sequence satisfying the condition of Theorem 1 . Theorem 1 gives the conclusion of Theorem 2.

Question 1. Is Theorem 2 true for every entire function of order less than $\frac{1}{2}$ without any kind of smoothness condition?

Remark 1. We note that (A) is implied by the following smooth condition: there exist a proximate order $\rho(r), \rho(r) \rightarrow \rho(r \rightarrow \infty)$ for some $\rho, 0 \leqq \rho<\frac{1}{2}$, and two constants $a, b$ such that

$$
0<a \leqq \varliminf_{r \rightarrow \infty} \frac{\log M(r, f)}{r^{\rho(r)}} \leqq \varlimsup_{r \rightarrow \infty} \frac{\log M(r, f)}{r^{\rho(r)}} \leqq b<+\infty
$$

(see Cartwright [3] for the definition and the properties of proximate order). Hence, for example, Theorem 2 is true for entire functions $f(z)$ which satisfy the condition

$$
\log M(r, f) \sim r^{\rho} \cdot \log ^{\rho_{1}} r \cdot \log _{2}^{\rho_{2}} r \cdots \log _{p}^{\rho_{p}} r \quad(r \rightarrow \infty)
$$

where $\log _{j} r=\log \left(\log _{j-1} r\right)$ and $\rho\left(0 \leqq \rho<\frac{1}{2}\right), \rho_{1}, \rho_{2}, \cdots, \rho_{p}$ are real numbers.

Next, we shall consider Julia directions of entire functions satisfying the smoothness condition (B).

A countable set of circles $C_{\nu}$ in $Z$ is said to form a slim set $S, S=$ $\bigcup_{\nu} C_{\nu}$, if the sum $\sum_{\nu} r_{\nu, k}$ of the radii $r_{\nu, k}$ of those circles $C_{\nu, k}$ intersecting the annulus $\left\{z: 2^{k} \leqq|z|<2^{k+1}\right\}$ is $o\left(2^{k}\right)(k \rightarrow \infty)$ i.e., 


$$
\varepsilon_{k} \rightarrow 0 \quad(k \rightarrow \infty) \text { for } \sum_{\nu} r_{\nu, k}=\varepsilon_{k} \cdot 2^{k}
$$

(see Anderson [1]).

LEMma 8. A slim set $S$ has the following properties:

(i) Each component of $S$ that intersect the set $\{z:|z|>N\}$ for $a$ sufficiently large number $N$ is contained in some annulus $R_{k}=\left\{z: 2^{k-1}<\right.$ $\left.|z|<2^{k+1}\right\}$,

(ii) Let $G_{k}$ be a component of $S$ contained in $R_{k}$. If we denote by $\theta_{k}$ the angle which $G_{k}$ subtends at the origin, then

$$
\theta_{k} \rightarrow 0 \quad(k \rightarrow \infty) .
$$

Proof. Evidently, (i) is true. If we denote $\theta_{\nu, k}$ the angle subtended at the origin by the circle $C_{\nu, k}$, we have

$$
\theta_{k} \leqq \sum_{\nu} \theta_{\nu, k-1}+\sum_{\nu} \theta_{\nu, k} \leqq \pi\left(\varepsilon_{k-1}+2 \cdot \varepsilon_{k}\right)
$$

Since $\varepsilon_{k} \rightarrow 0(k \rightarrow \infty)$, (ii) follows.

LEMma 9 (Anderson [1, Theorem 2]). Let $f(z)$ be an entire function for which $\log M(r, f)$ satisfies the condition (B). Then,

$$
\log |f(z)| \sim \log M(r, f) \quad(|z|=r \rightarrow \infty)
$$

outside a slim set $S_{f}$.

We deduce

THEOREM 3. Let $f(z)$ be an entire function for which $\log M(r, f)$ satisfies the condition (B). Then, the set of ray $\chi(\theta)$ for which $\theta$ is a limit point of the set

$$
E(f)=\left\{\arg z_{n}: f\left(z_{n}\right)=0\right\}
$$

is precisely the set of Julia directions of $f(z)$. In fact, if $\theta \in E(f), f(z)$ assumes every value without exception infinitely often in any sector containing $\chi(\theta)$. Otherwise $f(z)$ converges to $\infty$ as $|z| \rightarrow+\infty$ in some such sector and so assumes no value more than a finite number of times in this sector.

Proof. It is evident from Lemma 9 that $f(z)$ converges to $\infty$ as $|z| \rightarrow$ $+\infty$ in the sector which intersects a finite number of components of the $\operatorname{slim} \operatorname{set} S_{f}$.

Now, suppose that any sector containing $\chi(\theta)$ intersects an infinite 
number of components of $S_{f}$. Then, Lemma 8 shows that such sector contains an infinite number of components of $S_{f}$ completely. Here, we can easily see from Lemma 9 that for any fixed $M>0$, any component contained inside $R_{k}$, where $k$ is sufficiently large, contains at least one component of $F_{M}^{c}$, where $F_{M}^{c}$ denotes the complement of the set $\{z:|f(z)| \geqq M\}$. Thus, since such sector contains an infinite number of components of $F_{M}^{c}$, Rouche's theorem gives us the conclusion of Theorem 3.

QuEstion 2. A function satisfying (B) has order 0 (see Hayman [9, p. 130.]). As a natural generalization, we can consider the class of entire functions of order $\rho, 0 \leqq \rho<\frac{1}{2}$, satisfying the condition:

$$
\varlimsup_{r \rightarrow \infty} \frac{\log M(x \cdot r, f)}{x^{\rho} \cdot \log M(r, f)} \leqq 1
$$

for any $x, 1<x$.

Is the analogie of Theorem 3 true for this wider class, or for the still more general class satisfying the condition (A)?

The following example shows that Theorem 3 depends on the smoothness of growth of $M(r, f)$.

ExAmple. Let $\rho$ be any positive number. Take two sequences $\left\{a_{n}\right\}$, $\left\{b_{n}\right\}(n=1,2,3, \cdots)$ defined by

$$
a_{n}=c^{c^{n}}
$$

where $c=\left[1+1_{i}^{l} \rho\right]+1,[x]$ is the integral part of $x$, and

$$
\log ^{1+\rho} b_{n}=a_{n} \text {. }
$$

We define the entire function $f(z)$ by

$$
f(z)=\prod_{k=1}^{\infty}\left(1-\frac{z}{b_{k}}\right)^{a_{k}}
$$

This $f(z)$ has the following properties:

(a) Any $\chi(\theta),|\theta| \leqq \pi / 2$, is a Julia direction of $f(z)$, in spite of the fact that only $\theta=0$ is the limit point of the set $\left\{\arg z_{n}: f\left(z_{n}\right)=0\right\}$;

$$
\log M(r, f)=O\left(\log ^{2+\rho} r\right) \text {. }
$$

First of all, we shall show that

$$
\begin{aligned}
& f(z) \text { converges to } 0 \text { as }|z| \rightarrow+\infty \text { on the set } \\
& \bigcup_{n}\left\{z:\left|z-b_{n}\right|<c_{1} \cdot b_{n}\right\} \text { for any fixed } c_{1}, 0<c_{1}<1 .
\end{aligned}
$$


Decompose the product (4) into four subproducts $I_{i}(z)(i=1,2,3,4)$ :

$$
\begin{aligned}
I_{1}(z) & =\prod_{k=1}^{n-1}\left(\frac{z}{b_{k}}\right)^{a_{k}}, & I_{2}(z) & =\prod_{k=1}^{n-1}\left(\frac{b_{k}}{z}-1\right)^{a_{k}}, \\
I_{3}(z) & =\left(1-\frac{z}{b_{n}}\right)^{a_{n}}, & I_{4}(z) & =\prod_{k=n+1}^{\infty}\left(1-\frac{z}{b_{k}}\right)^{a_{k}} .
\end{aligned}
$$

We have to determine a upper bound of $I_{i}(z)(i=1,2,3,4)$ for any $z$, $\left|z-b_{n}\right|<c_{1} \cdot b_{n}$. First, we have

$$
\begin{aligned}
\left|I_{1}(z)\right| & \leqq \prod_{k=1}^{n-1}\left[\left(1+c_{1}\right) b_{n}\right]^{a_{k}}=\left(1+c_{1}\right)^{o(1) \cdot a_{n}} b_{n}^{(1+o(1)) \cdot a_{n-1}} \\
& =\left(1+c_{1}\right)^{o(1) \cdot a_{n}}\left(b_{n}^{a_{n-1} / a_{n}}\right)^{(1+o(1)) \cdot a_{n}}=(1+o(1))^{a_{n}} \quad(n \rightarrow \infty),
\end{aligned}
$$

because of the fact

$$
\sum_{k=1}^{n-1} a_{k}=o(1) \cdot a_{n} \quad(n \rightarrow \infty),
$$

and, since $c>1+1 / \rho$, we deduce

$$
b_{n}^{a_{n-1} / a_{n}} \rightarrow 1 \quad(n \rightarrow \infty) .
$$

Next, we have

$$
\begin{aligned}
\left|I_{2}(z)\right| & \leqq \prod_{k=1}^{n-1}\left(\left|\frac{b_{k}}{z}\right|+1\right)^{a_{k}} \leqq \prod_{k=1}^{n-1}\left(\frac{2-c_{1}}{1-c_{1}}\right)^{a_{k}}=\left(\frac{2-c_{1}}{1-c_{1}}\right)^{o(1) \cdot a_{n}} \\
& =(1+o(1))^{a_{n}}, \quad(n \rightarrow \infty)
\end{aligned}
$$

since

$$
\left|\frac{b_{k}}{z}\right| \leqq \frac{b^{k}}{\left(1-c_{1}\right) b_{n}}<\frac{1}{1-c_{1}} \quad(k=1,2,3, \cdots, n-1)
$$

For $I_{4}(z)$, we have

$$
\begin{aligned}
\left|I_{4}(z)\right| & \leqq \prod_{k=n+1}^{\infty}\left(1+\frac{\left(1+c_{1}\right) b_{n}}{b_{k}}\right)^{a_{k}} \leqq \exp \left[\left(1+c_{1}\right) b_{n} \cdot \prod_{k=n+1}^{\infty} \frac{a_{k}}{b_{k}}\right] \\
& =1+o(1) \quad(n \rightarrow \infty)
\end{aligned}
$$

by using the inequality

$$
1+x<e^{x} \quad(x>0)
$$

and

$$
b_{n} \cdot \prod_{k=n+1}^{\infty} \frac{a_{k}}{b_{k}} \rightarrow 0 \quad(n \rightarrow \infty) .
$$


Thus, we get

$$
|f(z)| \leqq[1+o(1)] \cdot\left[(1+o(1)) \cdot c_{1}\right]^{a_{n}} \quad(n \rightarrow \infty),
$$

which shows (5).

Next, we shall show that

$$
\begin{aligned}
& f(z) \text { converges to } \infty \text { as }|z| \rightarrow+\infty \text { on the sequence of circles } \\
& \left\{z:\left|z-b_{n}\right|=c_{2} \cdot b_{n}\right\} \text { for any fixed } c_{2}, c_{2}>1 .
\end{aligned}
$$

Decompose the product (4) into three subproducts $J_{j}(z)(j=1,2,3)$ :

$$
J_{1}(z)=\prod_{k=1}^{n-1}\left(1-\frac{z}{b_{k}}\right)^{a_{k}}, \quad J_{2}(z)=\left(1-\frac{z}{b_{n}}\right)^{a_{n}}, \quad J_{3}(z)=\prod_{k=n+1}^{\infty}\left(1-\frac{z}{b_{k}}\right)^{a_{k}}
$$

First of all, we have

$$
\left|J_{1}(z)\right| \geqq \prod_{k=1}^{n-1}\left(\left|\frac{z}{b_{k}}\right|-1\right)^{a_{k}} \geqq 1
$$

since

$$
\frac{|z|}{b_{k}} \geqq\left(c_{2}-1\right) \cdot \frac{b_{n}}{b_{k}} \geqq 2 \quad(k=1,2,3, \cdots, n-1)
$$

for sufficiently large $n$. Secondly, we have

$$
\begin{aligned}
\log \left|J_{3}(z)\right| & \geqq \sum_{k=n+1}^{\infty} a_{k} \cdot \log \left(1-\frac{\left(1+c_{2}\right) b_{n}}{b_{k}}\right) \\
& \geqq-2 \cdot \log 2 \cdot\left(1+c_{2}\right) \cdot b_{n} \cdot \sum_{k=n+1}^{\infty} \frac{a_{k}}{b_{k}}=o(1) \quad(n \rightarrow \infty),
\end{aligned}
$$

by using the inequality

$$
\log (1-x) \geqq-2 \cdot(\log 2) \cdot x \quad(0 \leqq x \leqq 1 / 2)
$$

and (7). Thus, we get

$$
|f(z)| \geqq(1-o(1)) \cdot c_{2}^{a_{n}}, \quad(n \rightarrow \infty)
$$

which shows (8).

Now, we can prove (a). Let $\theta$ be any fixed number satisfying $|\theta|<\pi / 2$ and denote by $\left\{z_{n}\right\}$ the point, other than the origin, where the ray $\chi(\theta)$ meets the circle $\left\{z:\left|z-b_{n}\right|=b_{n}\right\}$. Consider the sequence of functions

$$
f_{n}(z)=f\left(\left|z_{n}\right| \cdot z+z_{n}\right)
$$

and suppose that $\left\{f_{n}(z)\right\}$ is normal at $z=0$. Then, there is a $\delta, \delta>0$, 
such that $f(z)$ converges uniformly to some function $g(z)$ on the sequence of discs $D\left(z_{n}, \delta\right)$. If we take a $c_{1}$ in (5) and a $c_{2}$ in (8) such that

$$
1>c_{1}>1-2 \delta \cdot \cos \theta, \quad 1<c_{2}<1+2 \delta \cdot \cos \theta,
$$

then (5) and (8) show that $g(z) \equiv 0$ and $g(z) \equiv \infty$, respectively, which is a contradiction. Hence, we see that $\left\{f_{n}(z)\right\}$ is not normal at $z=0$. Now, Ostrowski [13, Satz 1 and p. 234] gives that $\chi(\theta),|\theta|<\pi / 2$, is Julia direction of $f(z)$. It is easy to see that $( \pm \pi / 2)$ is also a Julia direction of $f(z)$.

Next, we shall prove (b). For any $r, r \geqq b_{1}$, take an $n$ such that $b_{n} \leqq r<b_{n+1}$. Then, for the number $n(r, 1 / f)$ of zeros of $f(z)$ inside the circle $\{z:|z| \leqq r\}$, we have

$$
n\left(r, \frac{1}{f}\right)=\sum_{k=1}^{n} a_{k}=\sum_{k=1}^{n-1} a_{k}+a_{n}=(1+o(1)) \cdot a_{n} \leqq(1+o(1)) \cdot \log ^{1+\rho} r,
$$

from (6). Thus,

$$
r \cdot \int_{r}^{\infty} \frac{n(t, 1 / f)}{t^{2}} d t \leqq(1+o(1)) \cdot\left(\log ^{1+o} r\right) . \quad(r \rightarrow \infty)
$$

So we get

$$
\begin{aligned}
\log M(r, f) & =\log f(-r)=\int_{0}^{\infty} \log \left(1+\frac{r}{t}\right) d n\left(t, \frac{1}{f}\right) \\
& =r \cdot \int_{0}^{\infty} \frac{n(t, 1 / f)}{t(t+r)} d t \leqq \int_{0}^{r} \frac{n(t, 1 / f)}{t} d t+r \int_{r}^{\infty} \frac{n(t, 1 / f)}{t^{2}} d t \\
& =O\left(\log ^{2+\rho} r\right) .
\end{aligned}
$$

Remark 2. The property (5) shows that Lemma 9 holds only for the functions having some smoothness of growth of $M(r, f)$. From this fact, we can see that this example also satisfies

$$
\varlimsup_{r \rightarrow \infty} \frac{\log M(r, f)}{\log ^{2} r}=+\infty
$$

by the fact of Hayman [9, p. 143].

\section{§3. The set of Julia direction and growth of $M(r, f)$}

It is easily observed that the set of Julia directions of a transcendental entire function is a non-empty closed set. Polya [14] showed that for any given non-empty closed set $E$, there exists an entire function $f(z)$ of order $\infty$ having just $E$ as the set of Julia directions of $f(z)$. Anderson and 
Clunie [2, Theorem 1] also gave this sort of an example in the case $\rho=0$. Drasin and Weitsman [6, Theorem 1 and p. 209-210] constructed an example in the case $0<\rho \leqq 1 / 2$. But their construction depends on a general theorem of Levin [12, p. 95 and Chapter 2] and hence the condition $\rho>0$ is essential to show that a direction is a Julia direction.

The example in the following Theorem 4 generalizes the example of Anderson and Clunie [2] in the sense not only that it has order $\rho=0$ but also that it has an arbitrarily given growth subject to (B).

Lemma 10 (Valiron [15, p. 130], Edrei and Fuchs [7, Theorem 1]). Let $1(r)$ be a function

$$
1(r)=\text { constant }+\int_{r_{0}}^{r} \frac{\psi(t)}{t} d t, \quad\left(r \geqq r_{0}>0\right)
$$

where $\psi(t)$ is a non-negative, non-decreasing and unbounded function.

Assume further that

$$
\Lambda(r) \leqq r^{K}
$$

for some $K$ and all sufficiently large $r$.

Then, there exists an entire function $g(z)$ such that

$$
\log M(r, g) \sim \Lambda(r) \sim N\left(r, \frac{1}{g}\right) \quad(r \rightarrow \infty)
$$

where

$$
N\left(r, \frac{1}{g}\right)=\int_{0}^{r} \frac{n(t, 1 / g)-n(0,1 / g)}{t} d t+n\left(0, \frac{1}{g}\right) \cdot \log r .
$$

Lemma 11 (Hayman [9, Theorem 6]). Let $f(z)$ be an entire function. Then, $f(z)$ satisfies

$$
T(r, f) \sim T(2 r, f) \quad(r \rightarrow \infty)
$$

if and only if $f(z)$ has genus zero and further

$$
n\left(r, \frac{1}{f}\right)=o\left(N\left(r, \frac{1}{f}\right)\right) \quad(r \rightarrow \infty),
$$

where $T(r, f)$ denotes the characteristic function of $f(z)$.

Remark 3. That (B) is equivalent to (10) is easily seen from the inequality 


$$
T(r, f) \leqq \log ^{+} M(r, f) \leqq \frac{R+r}{R-r} T(r, f) \quad(0 \leqq r<R)
$$

(see [8, p. 18]).

THEOREM 4. Let $E$ be any non-empty closed set on $[0,2 \pi)$ and let $\Lambda(r)$ be a function given by

$$
\Lambda(r)=\text { constant }+\int_{r_{0}}^{r} \frac{\psi(t)}{t} d t \quad\left(r \geqq r_{0}>0\right)
$$

where $\psi(t)$ is a non-negative, non-decreasing and unbounded function.

Further, in the case

$$
\varlimsup_{r \rightarrow \infty} \frac{\Lambda(r)}{\log ^{2} r}=+\infty,
$$

we assume that

$$
\Lambda(2 r) \sim \Lambda(r) \quad(r \rightarrow \infty) .
$$

Then, there exists an entire function $f(z)$ such that

$$
\log M(r, f) \sim \Lambda(r) \quad(r \rightarrow \infty)
$$

and $E$ is precisely the set of Julia directions of $f(z)$.

Proof. First of all we remark by an argument of Hayman [9, p. 130] that (9) is satisfied for any positive $K$ if (11) holds.

Now, as in Edrei and Fuchs [7] we construct the function

$$
g(z)=\prod_{j=1}^{\infty}\left\{1+\left(\frac{z}{t_{j}}\right)^{q_{j}}\right\}
$$

such that

$$
\log M(r, g) \sim \Lambda(r) \sim N\left(r, \frac{1}{g}\right) \quad(r \rightarrow \infty)
$$

where $\left\{t_{j}\right\}$ and $\left\{q_{j}\right\}$ are the sequences chosen in [5, p. 388]. We take a countable dense subset $\left\{\theta_{1}, \theta_{2}, \theta_{3}, \cdots\right\}$ of $E$ and put

$$
z_{j, k}=t_{j} e^{i \theta_{k}} \quad\left(k=1,2,3, \cdots, q_{j} ; j=1,2,3, \cdots\right) .
$$

We define the required function $f(z)$ by

$$
f(z)=\prod_{j=1}^{\infty} \prod_{k=1}^{k=q_{j}}\left(1-\frac{z}{z_{j, k}}\right) .
$$


First, in the case

$$
\varlimsup_{r \rightarrow \infty} \frac{\Lambda(r)}{\log ^{2} r}=+\infty,
$$

we have from (11) and (12) that

$$
\log M(2 r, g) \sim \log M(r, g) \quad(r \rightarrow \infty) .
$$

Hence, by Lemma 11 and Remark 3,

$$
n\left(r, \frac{1}{g}\right)=o\left(N\left(r, \frac{1}{g}\right)\right) \quad(r \rightarrow \infty)
$$

and $g(z)$ has genus zero. Again from Lemma 11, Remark 3, (12) and the fact of Hayman [9, p. 133],

(13) $\log M(2 r, f) \sim \log M(r, f) \sim N\left(r, \frac{1}{f}\right)=N\left(r, \frac{1}{g}\right) \sim \Lambda(r) \quad(r \rightarrow \infty)$.

Thus, this $f(z)$ satisfies

$$
\log M(r, f) \sim \Lambda(r) \quad(r \rightarrow \infty) .
$$

In the case

$$
\varlimsup_{r \rightarrow \infty} \frac{\Lambda(r)}{\log ^{2} r}<+\infty
$$

from (12) and Hayman [9, p. 143],

$$
n\left(r, \frac{1}{g}\right)=o\left(N\left(r, \frac{1}{g}\right)\right) \quad(r \rightarrow \infty)
$$

and hence

$$
n\left(r, \frac{1}{f}\right)=o\left(N\left(r, \frac{1}{f}\right)\right) \quad(r \rightarrow \infty)
$$

Thus by the same argument, this $f(z)$ satisfies

$$
\log M(2 r, f) \sim \log M(r, f) \sim \Lambda(r) \quad(r \rightarrow \infty) .
$$

Now, it is easily observed from (13) and (14) and Theorem 3 that $E$ is precisely the set of Julia directions of $f(z)$. 


\section{REFERENCES}

[1] J. M. Anderson, Asymptotic values of meromorphic functions of smooth growth, Glasgow Math. J., 20 (1979), 155-162.

[2] J. M. Anderson and J. Clunie, Entire functions of finite order and lines of Julia, Math. Z., 112 (1969), 59-73.

[ 3 ] M. L. Cartwright, Integral functions, Cambridge, 1956.

[ 4 ] J. Clunie and W. K. Hayman, The spherical derivative of integral and meormorphic functions, Comment. Math. Helv., 40 (1966), 117-148.

[ 5 ] A. Denjoy, Sur un théorème de Wiman, C. R. Acad. Sci., 193 (1931), 828-830.

[ 6 ] D. Drasin and A. Weitsman, On the Julia directions and Borel directions of entire functions, Proc. London Math. Soc., 32 (1976), 199-212.

[ 7 ] A. Edrei and W. H. J. Fuchs, Entire and meromorphic functions with asymptotically prescribed characteristic, Canad. J. Math., 17 (1965), 383-395.

[ 8 ] W. K. Hayman, Meromorphic functions, Oxford, 1964.

[9] - On Iversen's theorem for meromorphic functions with few poles, Acta Math., 141 (1978), 115-145.

[10] B. Kjellberg, On certain integral and harmonic functions, Upsala, 1948 (Dissertation).

[11] O. Lehto, The spherical derivative of a meromorphic function in the neighborhood of an isolated essential singularity, Comment. Math. Helv., 33 (1959), 196-205.

[12] B. Levin, Distribution of the zeros of entire functions, Amer. Math. Soc. Transl., 5 (1964).

[13] A. Ostrowski, Über folgen analytischer Funktionen und einige Verschärfungen des Picardschen Satzes, Math. Z., 24 (1926), 215-258.

[14] G. Polya, Untersuchungen über Lücken und Singularitäten von Potenzreihen, Math. Z., 29 (1929), 549-640.

[15] G. Valiron, Sur les fonctions entières d'ordre fini et d'ordre nul, et en particulier les fonctions à correspondance régulière, Ann. Fac. Sci. Toulouse, 5 (1913), 117208.

Department of Mathematics

Faculty of Science

Chiba University

Yayoi-cho, Chiba 260 Japan 